\title{
THE CONSTRUCTION OF THE FAST RESISTIVE BOLOMETER FOR A SXR MEASUREMENT ON THE GIT-12 FACILITY
}

\author{
Jakub Cikhardt $^{a, *}$, Daniel Klír ${ }^{a}$, Pavel Kubeš ${ }^{a}$, Josef Kravárik $^{a}$, \\ Karel ŘezÁČ ${ }^{a}$, OndŘej Š́́la ${ }^{a}$, AleXander V. Shishlov ${ }^{b}$, \\ Alexey Yu. LABETSKY ${ }^{b}$ \\ a Department of Physics, Faculty of Electrical Engineering, Czech Technical University in Prague, Czech Republic \\ ${ }^{b}$ Institute of High Current Electronics, Siberian Branch of the Russian Academy of Sciences in Tomsk, Russian \\ Federation \\ * corresponding author: cikhajak@fel.cvut.cz
}

\begin{abstract}
A lot of kinds of instruments are used for the SXR measurement at pulsed power facilities, but most of them are difficult to calibrate absolutely. For the determination of the energy of SXR radiated by the discharge on Z-pinches, it is possible to use the bolometer which can be calibrated analytically. The bolometer can be constructed with the sufficient sensitivity and, at the same time, with the time resolution in the order of nanoseconds. This bolometer was designed and constructed for the measurement on the 5 MA facility GIT-12 at the Institute of High Current Electronics (IHCE) of the Siberian Branch Russian Academy of Sciences in Tomsk. The experiments on GIT-12 with the neon and deuterium gas-puff load were diagnosed by the copper bolometer with the time resolution of $4 \mathrm{~ns}$ and the sensitivity of $12 \mathrm{~V} \mathrm{~cm}^{2} \mathrm{~J}^{-1}$.
\end{abstract}

KeYwords: bolometers, X-rays, Z-pinches, plasma diagnostics.

\section{INTRODUCTION}

The ohmic bolometer is based on the principle of transformation of radiated energy into thermal energy. It leads to the change of the resistance according to the well known formula

$$
R=R_{0}(1+\alpha \Delta \vartheta)
$$

where $R_{0}$ is the initial resistance, $\alpha$ is the thermal coefficient of the resistivity and $\Delta \vartheta$ is the change of the temperature. In the case of metallic bolometers, the coefficient $\alpha$ can be considered as a positive constant, thus the dependence of the resistivity on the temperature is linear.

The parameters and design are dependent on the application of a bolometer. Bolometers are used in microwave technology, pyrometry, astronomy and in plasma diagnostics. The bolometer described in this paper is designed for measurement of the pulses of SXRs produced by a short lived plasma generated by pulse power generators especially large Z-pinches. The experiments with this bolometer were performed with triple shell neon and deuterium gas-puffs. During the stagnation of the dense neon gas-puff, the most of kinetic energy of the Z-pinch is radiating in neon K-shell lines $(h \nu \cong 0.9 \div 1.4 \mathrm{keV})$ [4]. In case of the deuterium, the main component of the radiation is the continuous bremsstrahlung.

In this case the sensitive element of the bolometer is a very thin metal stripe. Dimensions and material of the stripe were determined with regard to the requirement for the bolometer:
- sufficient sensitivity which is constant on wide range of the photon energy,

- time resolution in the order of nanoseconds,

- ability to work in environments with extreme electromagnetic noise.

\section{Parameters Determination}

\subsection{Sensitivity}

The sensitivity $s$ of this detector is determined as a ratio between the change of the output voltage and the fluence $\varrho$ at the point where the bolometer is placed,

$$
s=\frac{\Delta U}{\Delta Q / S}=\frac{\Delta U}{\Delta \varrho},
$$

where $S$ is the surface of the bolometer sensitive element. Using Eq. 1 and another well known physical formulas we obtain the sensitivity determined by the dimensions of the sensitive element, material constants and bolometer current,

$$
s=\rho_{\mathrm{E}} l \alpha \frac{1}{d^{2} C \rho w} I,
$$

where $\rho_{\mathrm{E}}$ is resistivity, $\alpha$ is the thermal coefficient of the resistivity, $C$ is the specific heat, $\rho$ is the density of the material, $l, d$ and $w$ are length, thickness and width of the sensitive element respectively and $I$ is the current of the bolometer.

\subsection{RANGE OF PHOTON ENERGIES}

The photon energies which can be measured with the bolometer are determined by the radiation absorption. For our purpose the data from [3] were used. 

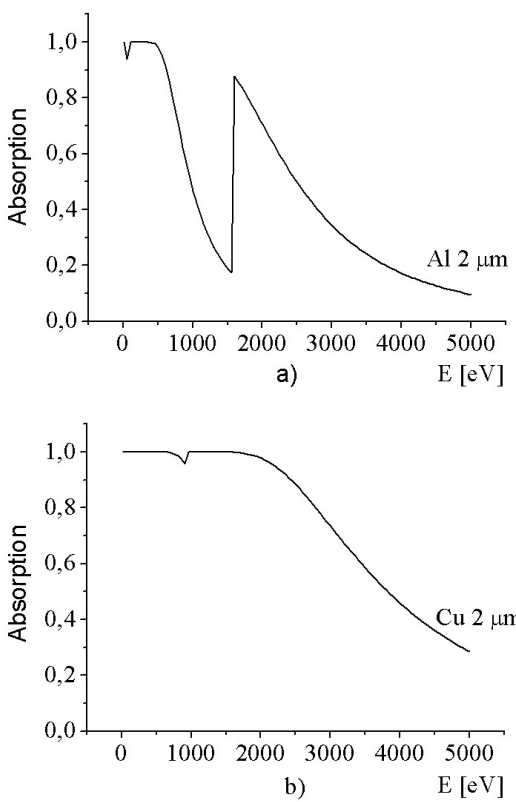

FiguRE 1. The absorption characteristics of 2 micron metal foils: a) aluminium foil, b) copper foil.

In the Fig. 1 the absorption characteristics of the aluminium and copper of 2 micron thickness are shown as examples.

In Fig. 17a, the absorption of the aluminium foil has a minimum at about $1.6 \mathrm{keV}$. This minimum corresponds to the $\mathrm{K}$-shell edge of the aluminium [8]. If the bolometer is made of this foil, the detector will be selective. The absorption characteristic of 2 micron thick copper foil can be seen in the Fig. 1 b. This characteristic is much more "smooth" than the characteristic of the aluminium. For our application, a nonselective detector is needed in the range up to $2 \mathrm{keV}$, therefore the copper foil was chosen. If the photon energy range is considered as a region where the sensitivity is not less than $1 / \sqrt{2}$ of the maximal sensitivity, the energy range of the bolometer made of 2 micron thick copper is up to $3 \mathrm{keV}$.

\subsection{Time RESOLUTION}

The time resolution of bolometers is defined by the characteristic time needed to equilibration of the temperature in the sensitive element. If the front side of the metal foil is irradiated by a short SXR pulse, the absorbed energy will have the exponential dependence on the depth inside the foil [1]. The energy of SXR absorbed in the material is transformed into the thermal energy. Therefore we can consider that the dependence of temperature on the depth inside the metal foil is also exponential,

$$
\vartheta(x, 0)=\vartheta(0,0) \mathrm{e}^{-\gamma \rho x},
$$

where $x$ is the distance from the irradiated surface of the foil and $\gamma$ is the absorption coefficient of material. We also consider that there is no heat transfer

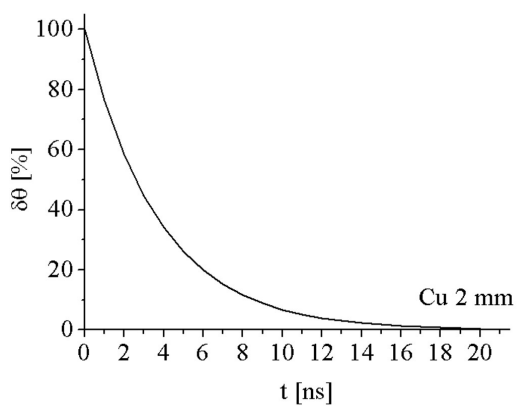

Figure 2. The time dependence of the temperature difference of the sides of the 2 microns copper foil.

between the sensitive element and surroundings because in our detector it is usually placed in vacuum. Therefore we can write

$$
\frac{\partial \vartheta(0, t)}{\partial x}=\frac{\partial \vartheta(d, t)}{\partial x}=0
$$

Using the conditions Eq. 4 and Eq. 5 in the onedimensional heat transfer equation [6]

$$
\frac{\partial^{2} \vartheta(x, t)}{\partial x^{2}}=\frac{1}{\chi} \frac{\partial \vartheta(x, t)}{\partial t}
$$

we obtain solution

$$
\begin{aligned}
& \vartheta(x, t)=\frac{\vartheta_{0}}{\gamma \rho d}\left(1-\mathrm{e}^{-\gamma \rho d}\right)+ \\
& +\frac{2 \vartheta_{0}}{d} \sum_{n=1}^{\infty} \frac{1-(-1) \mathrm{e}^{-\gamma \rho d}}{\left(\frac{\pi n}{\gamma \rho d}\right)^{2}+1} \cos \left(\frac{\pi n x}{d}\right) \mathrm{e}^{-\left(\frac{\pi n}{d}\right)^{2} \chi t}
\end{aligned}
$$

where $\vartheta_{0}=\vartheta(0,0)$ and $\chi$ is thermal diffusivity. For determination of the characteristic time, it is useful to study the thermal difference between both sides of the foil: $\vartheta(0, t)-\vartheta(d, t)$. The characteristic time of bolometer can be defined as the time at which the temperature difference drops at $1 / \mathrm{e}$ of the maximum of value. If this exponential process is equal to $1 / \mathrm{e}$ we consider that temperature in the foil is equilibrated. This temperature difference is plotted in the Fig. 2

The characteristic time of the bolometer with sensitivity element made of 2 microns copper foil is $4 \mathrm{~ns}$.

The most important properties of the designed bolometer are shown in the Tab. 1 .

\section{Construction}

\subsection{BOLOMETRIC DETECTOR}

Cross-section of the bolometric detector is shown in the Fig. 3 .

The foil is fixed by mechanical clamps. Because the resistance of the foil is low $\left(R_{0}=0.126 \Omega\right)$ the fourclamp method was used for connection to the electric circuit. Structure of the sensitive element and the surrounding circuits is symmetrical in order to achieve 


\begin{tabular}{|c|c|}
\hline Property & Value \\
\hline Material & copper \\
\hline Thickness & 2 microns \\
\hline Width & $2 \mathrm{~mm}$ \\
\hline Length & $30 \mathrm{~mm}$ \\
\hline Initial resistance & $(0.126 \Omega)$ \\
\hline Time resolution & $4 \mathrm{~ns}^{2} \mathrm{~J}^{-1}$ \\
\hline Sensitivity & $12.43 \mathrm{~V} \mathrm{~cm}^{2}$ \\
\hline
\end{tabular}

TABLE 1. The properties of the designed bolometer.

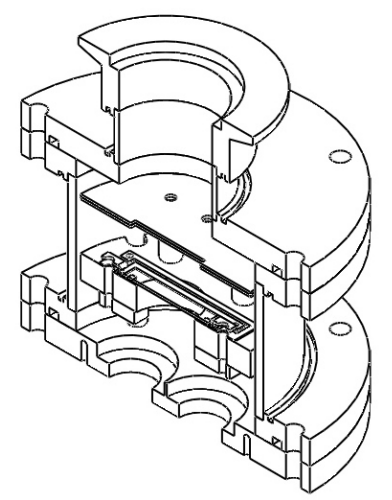

Figure 3. Cross-section of the bolometric detector.

a lower inductance. The screen with aperture is placed above the sensitive element. This screen protects the passive parts of the detectors against the irradiation and it allows to place a filter between the sensitive element and the source of radiation.

\subsection{POWER SUPPLY}

To achieve the required sensitivity, it is necessary to supply the bolometer with a current of least $10 \mathrm{~A}$. To avoid melting of the thin foil it is necessary to supply the bolometer with a short current pulse and at the same time the power supply pulse must be longer than the duration of the SXR pulse. We choose $10 \mu \mathrm{s}$ length of the supply pulse. The bolometer changes resistance because of temperature change. Therefore we need to know bolometer voltage and at the same time the bolometer current. We constructed MOS-FET pulsed power supply with a defined output current, thus we need to register the bolometer output voltage only. The comparison of the measured and simulated dependence of the power supply current on the load resistance is shown in the Fig. 4

\section{EXPERIMENTS ON GIT-12 FACILITY}

The bolometer was used during Z-pinch experiments on the GIT-12 facility at the Institute of High Current Electronics (IHCE) of the Siberian Branch Russian Academy of Sciences in Tomsk. The GIT-12 is large pulsed power generator with 12 Marx generators. The peak current of this facility reaches $5 \mathrm{MA}$, the rise time is $200 \mathrm{~ns}$ with plasma opening switches

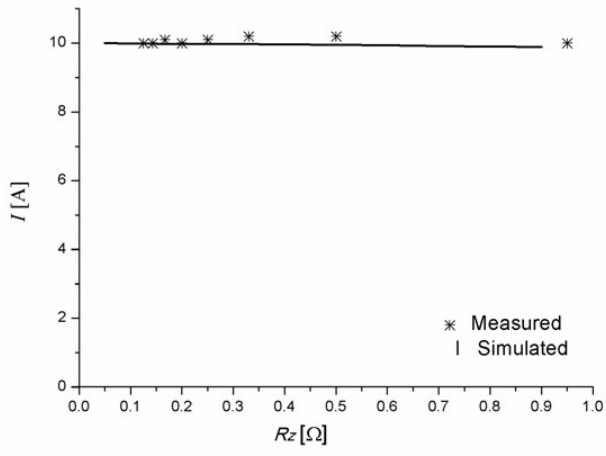

FiguRE 4. The comparison of the measured and simulated dependence of the power supply current on the load resistance.

(POS) and $1400 \mathrm{~ns}$ without POS, total energy stored in capacitors is $2.6 \mathrm{MJ}$.

The load of these experiments was a triple shell gas-puff of $160 \mathrm{~mm}, 80 \mathrm{~mm}$ and $30 \mathrm{~mm}$ diameters of the individual nozzles. Neon, deuterium $\left(\mathrm{D}_{2}\right)$, argon and their combination of this were used as filling gases for the gas-puff. The typical length of the gas column during these experiments was about $2 \mathrm{~cm}$. The bolometer was placed in $2 \mathrm{~m}$ distance from the discharge without any filter.

The typical detector output signal, namely the shot with neon (see shot no. 1 in Tab. 2) is plotted in the Fig. 5 The signal without a SXR pulse is plotted also for the comparison. The power supply current of the bolometer is starting to rise at the time $6 \mu \mathrm{s}$ and at the time $8 \mu \mathrm{s}$ is fully stabilized. The electromagnetic noise at the time around $10 \mu \mathrm{s}$ is caused by ignition of the spark-gaps in the Marx generators. The irradiation of the bolometer occurs at time of $12 \mu \mathrm{s}$, when the significant voltage step is observed. The magnitude of this voltage step corresponds to $50 \mathrm{~kJ}$ of radiated energy during implosion of the gas-puff. Other measured values including the shots in the deuterium and combination of the deuterium and neon, or argon are shown in Tab. 2 All these shots were carried out with the triple shell gas-puffs. The notation A-B-C means gas $\mathrm{A}$ in outer the shell, gas $\mathrm{B}$ in the middle shell and gas $\mathrm{C}$ in the inner shell.

Results of these shots are shown in the Tab. 2

\subsection{Discussion OF THE EXPERIMENTAL RESULTS}

It is seen in Tab. 2 that the radiation in shot with the deuterium was lower. Thus more of the kinetic energy of the Z-pinch is transformed to the thermal energy.

It is not easy to theoretically calculate the kinetic energy of the Z-pinch exactly. For a rough estimation of the kinetic energy of the Z-pinch can be used the formula [8]

$$
E_{\mathrm{k}}=2.3 I_{\mathrm{m}}^{2} l(\mathrm{~kJ}),
$$

where $I_{\mathrm{m}}$ is the maximum of the current peak in MA and $l$ is the length of the discharge in $\mathrm{cm}$. 


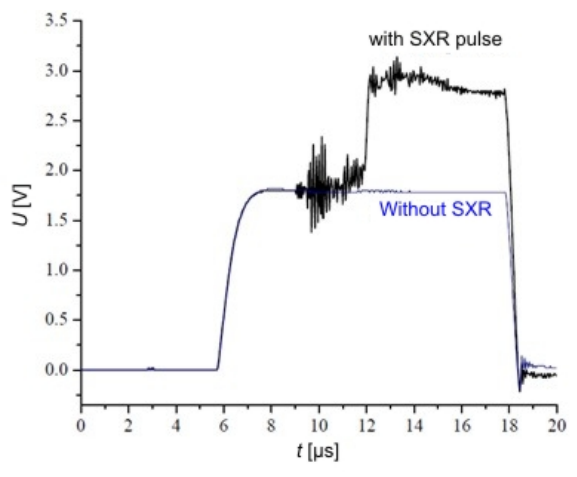

FiguRE 5. The signal of the bolometric detector during the discharge in comparison with detector's signal without the irradiation.

\begin{tabular}{|c|c|c|c|}
\hline $\begin{array}{c}\text { Shot } \\
\text { No. }\end{array}$ & $\begin{array}{c}\text { Current } \\
\text { (MA) }\end{array}$ & $\begin{array}{c}\text { Triple shell } \\
\text { Gas-puff }\end{array}$ & $\begin{array}{c}\text { SXR energy } \\
(\mathbf{k J})\end{array}$ \\
\hline 1 & 3.73 & $\mathrm{Ne}-\mathrm{Ne}-\mathrm{Ne}$ & 50 \\
\hline 2 & 3.55 & $\mathrm{D}_{2}-\mathrm{D}_{2}-\mathrm{D}_{2}$ & 13 \\
\hline 3 & 3.64 & $\mathrm{D}_{2}-\mathrm{D}_{2}-\mathrm{D}_{2}$ & 22 \\
\hline 4 & 2.58 & $\mathrm{D}_{2}-\mathrm{D}_{2}-\mathrm{D}_{2}$ & 21 \\
\hline 5 & 3.60 & $\mathrm{Ne}-\mathrm{D}_{2}-\mathrm{D}_{2}$ & 28 \\
\hline 6 & 3.36 & $\mathrm{Ne}-\mathrm{D}_{2}-\mathrm{D}_{2}$ & 23 \\
\hline 7 & 3.36 & $\mathrm{Ne}-\mathrm{D}_{2}-\mathrm{D}_{2}$ & 26 \\
\hline 8 & 3.10 & $\mathrm{Ne}-\mathrm{D}_{2}-\mathrm{D}_{2}$ & 18 \\
\hline 9 & 3.28 & $\mathrm{Ne}-\mathrm{D}_{2}-\mathrm{D}_{2}$ & 24 \\
\hline 10 & 3.66 & $\mathrm{Ar}-\mathrm{D}_{2}-\mathrm{D}_{2}$ & 14 \\
\hline 11 & 2.16 & $\mathrm{D}_{2}-\mathrm{D}_{2}-\mathrm{D}_{2}$ & 27 \\
\hline
\end{tabular}

TABLE 2. Results of the SXR measurement at the experiments on GIT-12 facility.

Substituting values from shot no. 1 we obtain the kinetic energy about $64 \mathrm{~kJ}$. It corresponds to measured values and to the assumption that the most of kinetic energy of the neon gas-puff is changed to the energy of the radiation.

The temperature of the bolometer at the steady state can be determined by the well known formula $\Delta \vartheta=\Delta Q /(\mathrm{Cm})$, where $\Delta Q$ is an absorbed energy, $C$ is a specific heat capacity and $m$ is the mass of the bolometer foil. Substituting the parameters of the bolometer and radiated energy of $50 \mathrm{~kJ}$ from the shot no. 1, we obtain the change of the temperature of $144 \mathrm{~K}$. If the initial temperature of the bolometer was $293 \mathrm{~K}$, the temperature after irradiation is $437 \mathrm{~K}$. So the melting point of the copper at $1357 \mathrm{~K}$ was not achieved.

The accuracy of this measurement is decreased by several error factors. The most significant factor is electromagnetic noise. For example the RMS value of this noise in shot no. 1 achieved to $7 \%$ of the useful signal. Including the noise, uncertainty of the stability of the power supply current and uncertainty of the dimensions of the bolometer foil, the total uncertainty of the measured radiated energy is about $10 \%$.

\section{Conclusion}

The bolometric detector for pulse SXR measurement was designed and constructed. The experiments on the GIT-12 facility was performed with this detector. The sensitivity, noise immunity and uncertainty of the detector is sufficient for the measurement at the terawatt generator GIT-12. Amount of energies were measured in the range from $13 \mathrm{~kJ}$ to $50 \mathrm{~kJ}$. It depended on the current peak and gas of the load and its amount.

The measurements confirmed the assumption that the discharges in the deuterium produced less amount of the radiated energy of SXRs than the discharges in gases with higher atomic number. However the energy of SXRs radiated during the discharge in deuterium gas-puff was surprisingly high. Probably it is the radiation of the material of electrodes. The measured values corresponds to the theoretical estimation of the kinetic energy of the gas-puff. Because the amount of the radiated energy carries the information about the real kinetic energy of the gas-puff, it can be used for the gas-puff optimization as a scale.

For determination of the dependences of the amount of the radiated energy on the gas-puff parameters and for verification of the detector's time resolution we need more experimental shots which are scheduled on spring 2013.

\section{ACKNOWLEDGEMENTS}

This work was supported by the Research Program MSMT No. LA08024, ME09087 and GACR P205/12/0454, IAEA RC 14817 and CTU SGS 10/266/OHK3/3T/13.

\section{REFERENCES}

[1] D. Attwood. Soft X-rays and extreme ultraviolet radiation: Principles and applications. Canbridge University press, 1999.

[2] J. Cikhardt. Konstrukce rychleho bolometru pro mereni intenzity impulsniho mekkeho rentgenového zareni. FEE CTU, Prague, 2012. Diploma thesis.

[3] E. M. Gullikson. Filter transmission. http: //henke.lbl.gov/optical_constants/filter2.html The Center for X-Ray Optics.

[4] A. Yu. Labetsky, V. A. Kokshenev, N. E. Kurmaev, et al. Study of the current-sheath formation during the implosion of multishell gas puffs. Plasma Physics Reports 34(3):228-238, 2008.

[5] M. A. Liberman, J. S. De Groot, A. Toor, R. B. Spielman. X-ray data booklet. Berkley. Lawrence Berkley National Laboratory University of California, 2009. 3rd edition.

[6] J. H. Lienhard IV, J. H. Lienhard V. A Heat Transfer Textbook. Phlogiston Press, Cambridge, Massachusetts, 2008. 3rd edition.

[7] R. B. Spielman, C. Deeney, D. L. Fehl, et al. Fast resistive bolometer. Tech. Rep. SAND98-1987, Sandia National Laboratories, 1998.

[8] A. Thompson, et al. X-ray data booklet. Lawrence Berkley National Laboratory University of California, Berkley, 2009. 3rd edition. 